\title{
BMJ Open Low-dose aspirin for preventing intrauterine growth restriction and pre- eclampsia in sickle cell pregnancy (PIPSICKLE): a randomised controlled trial (study protocol)
}

Bosede Bukola Afolabi (10 , ${ }^{1}$ Ochuwa Adiketu Babah, ${ }^{1}$ Titilope Adenike Adeyemo, ${ }^{2}$ Oluwakemi Ololade Odukoya, ${ }^{3}$ Chinyere Veronica Ezeaka, ${ }^{4}$ Obinyo Nwaiwu, ${ }^{5}$ Yusuf Abisowo Oshodi, ${ }^{6}$ Babatunde A Ogunnaike ${ }^{7}$

To cite: Afolabi BB, Babah OA, Adeyemo TA, et al. Lowdose aspirin for preventing intrauterine growth restriction and pre-eclampsia in sickle cell pregnancy (PIPSICKLE): a randomised controlled trial (study protocol). BMJ Open 2021;11:e047949. doi:10.1136/ bmjopen-2020-047949

- Prepublication history for this paper is available online. To view these files, please visit the journal online (http://dx.doi. org/10.1136/bmjopen-2020047949).

Received 12 December 2020 Accepted 30 July 2021

A) Check for updates

(c) Author(s) (or their employer(s)) 2021. Re-use permitted under CC BY-NC. No commercial re-use. See rights and permissions. Published by BMJ.

For numbered affiliations see end of article.

Correspondence to Professor Bosede Bukola Afolabi;

bbafolabi@unilag.edu.ng

\section{ABSTRACT}

Introduction Pregnancy in sickle cell disease is fraught with many complications including preeclampsia (PE) and intrauterine growth restriction (IUGR). Previously, we found an abnormality in prostacyclin-thromboxane ratio in sickle cell pregnant women, a situation that is also found in non-sickle pregnancies with PE and unexplained IUGR. Low-dose aspirin (LDA) has been shown to reduce the incidence of PE and IUGR in high-risk women by reducing the vasoconstrictor thromboxane while sparing prostacyclin, in effect 'correcting' the ratio. It has been found to be safe for use in pregnancy but has not been tested in sickle cell pregnancy. We hypothesise that LDA will reduce the incidence of IUGR and PE in pregnant haemoglobin SS (HbSS) and haemoglobin SC (HbSC) women.

Methods and analysis This is a multisite, double blind, randomised controlled trial, comparing a daily dose of $100 \mathrm{mg}$ aspirin to placebo, from 12 to 16 weeks' gestation until 36 weeks, in Lagos state, Nigeria. Four hundred and seventy-six eligible pregnant $\mathrm{HbSS}$ and $\mathrm{HbSC}$ women will be recruited consecutively, randomly assigned to either group and followed from recruitment until delivery. The primary outcome will be the incidence of birth weight below 10th centile for gestational age on INTERGROWTH 21 birth weight charts, or incidence of miscarriage or perinatal death. Secondary outcomes will include PE, maternal death, preterm delivery, perinatal death, number of crises, need for blood transfusion and complications such as infections and placental abruption. Analysis will be by intention to treat and the main treatment effects will be quantified by relative risk with $95 \% \mathrm{Cl}$, at a $5 \%$ significance level.

Ethical approval Ethical approval has been granted by the Health Research and Ethics committees of the recruiting hospitals and the National Health Research and Ethics Committee. Study findings will be presented at conferences and published appropriately.

Trail registration number PACTR202001787519553; Pre-results.

\section{Strengths and limitations of this study}

- Multisite randomised, controlled parallel superiority trial, stratified by site.

Double-blind study.

- Largest number of prospectively studied pregnant women with sickle cell disease.

- Care of the women may vary between sites and affect some of the outcomes.

\section{INTRODUCTION}

\section{Background and rationale}

Nigeria has the highest number of people living with sickle cell disease (SCD) in the world, amounting to $2 \%-3 \%$ of the population. ${ }^{1}$ SCD is a haemoglobinopathy fraught with complications as a result of vasoocclusion, thrombosis and chronic anaemia. The red blood cells become abnormal and fragile when deoxygenated, forming polymers and adhering to vascular endothelial tissue with resultant obstruction of the vessels and haemolysis. ${ }^{2}{ }^{3}$ These phenomena lead to the various clinical manifestations, which include splenic sequestration, susceptibility to infections, stroke, bone pain crises and acute chest syndrome. Vasoconstriction from nitric oxide depletion has also been postulated to be a pathophysiological mechanism for some of the SCD adverse effects such as pulmonary hypertension and stroke. ${ }^{4}$

Over $50 \%$ of women with SCD now achieve pregnancy due to increased survival into adulthood but they have a high incidence of complications during pregnancy including pre-eclampsia $(\mathrm{PE})$ and intrauterine growth restriction (IUGR), infections, acute chest syndrome and more frequent crises. ${ }^{5-7} \mathrm{~A}$ 
number of pregnant women with SCD are thus more likely to die in pregnancy especially in low resource settings like ours $^{7}$; pregnancy-related studies in them should therefore be a priority. Even when these women do not die, a large proportion of them end up as 'maternal near miss', meaning they would have died but for prompt and appropriate intervention. ${ }^{8}$

\section{Choice of comparator}

Previously, we found a reversal in prostacyclin-thromboxane ratio in pregnant haemoglobin SS (HbSS) women, ${ }^{9}$ a situation that is also found in non-sickle pregnancies with PE and unexplained IUGR. ${ }^{10}$ Low-dose aspirin (LDA) has been shown to reduce the incidence of PE and IUGR in high-risk women due to its reduction of vasoconstrictor thromboxane while sparing prostacyclin, in effect correcting the ratio. ${ }^{11}$ It has been found to be safe for use in pregnancy ${ }^{12}$ and has been suggested for use in sickle cell pregnant women, ${ }^{13}$ but all the studies were conducted in non-SCD pregnant women. To the best of our knowledge, there are no studies in the scientific literature evaluating the role of $\mathrm{LDA}$ in prevention of $\mathrm{PE}$ and IUGR in pregnant SCD women. As women with SCD have such a high incidence of morbidity and mortality in pregnancy, the results of this study could potentially save their lives and that of their babies. We chose placebo as our comparator because there is no other known drug that is already in use that has the possible chemical potential to effect a reversal of prostacyclin-thromboxane ratio.

The objectives of this study are:

1. To determine the effect of the use of LDA during pregnancy in HbSS and haemoglobin SC (HbSC) women on :

a. The risk of IUGR, perinatal death or miscarriage.

b. The risk of other maternal complications including PE, preterm delivery, number of vaso-occlusive crises, need for blood transfusion, urinary tract infections, respiratory tract infections, acute chest syndrome, retained placenta, placental abruption and vaginal bleeding.

2. To build capacity in conducting randomised controlled clinical trials especially for junior faculty in Nigeria who will be involved in the research.

\section{Trial design}

We propose the multicentre, double blind, superiority randomised controlled trial presented here (figure 1).

\section{METHODS AND ANALYSIS}

This clinical trial commenced on 1 July 2020 and the scheduled duration is 2 years. The coordinating centre is domiciled at the Department of Obstetrics and Gynaecology, College of Medicine, University of Lagos, Nigeria. The primary study site is Lagos University Teaching Hospital, Idi-Araba, Lagos, Nigeria, its affiliated teaching hospital. Fourteen other public hospitals were purposively selected as study sites because they have specialists

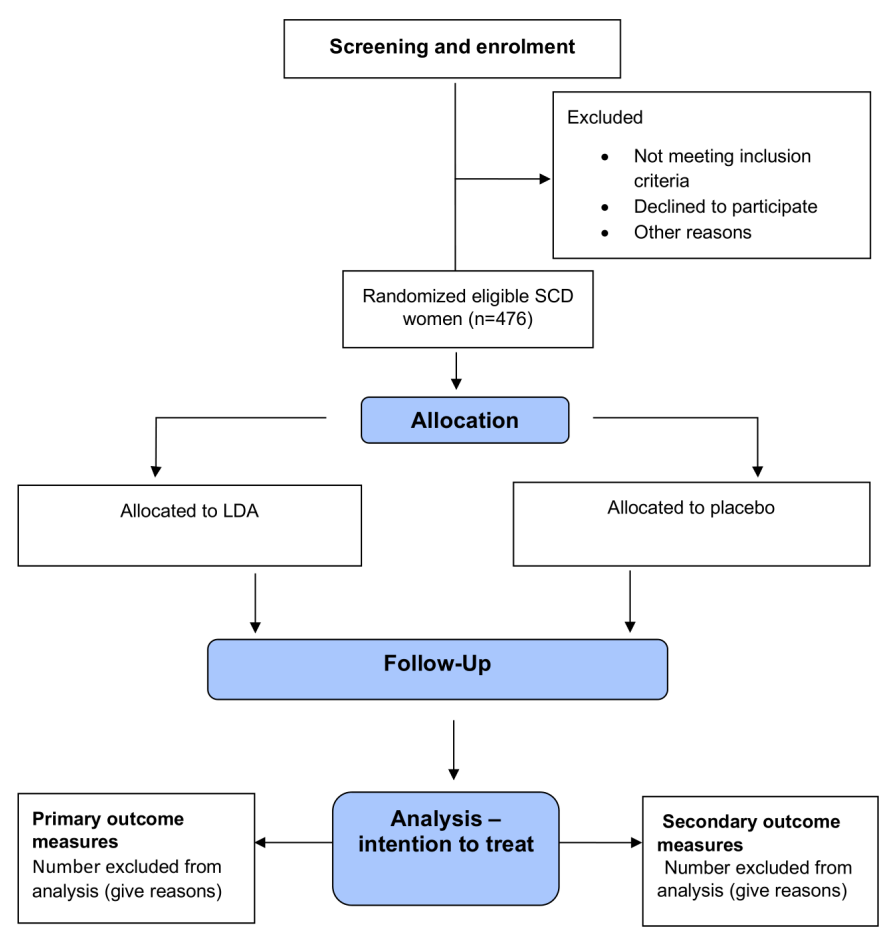

Figure 1 Trial flow chart. LDA, low-dose aspirin; SCD, sickle cell disease.

that have special interest in sickle cell pregnancy and/or a relatively high number of pregnant women with SCD. This is because the incidence of sickle cell pregnancy within the general pregnant population is relatively low, approximately $1 \%$ of all pregnancies. ${ }^{1415}$

\section{Eligibility criteria}

The inclusion criteria are:

1. Age 18 years and above.

2. Singleton fetus.

3. Women whose genotypes are HbSS or HbSC.

4. 16 weeks gestation or less at recruitment, estimated from the last menstrual period or by an early ultrasound scan.

Excluded from the study are:

1. Women with associated medical conditions in pregnancy for example, HIV infection, diabetes mellitus, chronic hypertension, renal disease, sickle nephropathy.

2. Multiple pregnancy.

3. Hypersensitivity to aspirin.

4. History of blood transfusion in the last 3 months.

Each study site is manned by a trained site investigator who is an obstetrician/gynaecologist, and a trained midwife.

\section{Interventions}

The intervention is LDA at a dose of $100 \mathrm{mg}$ daily. The comparison group takes a placebo tablet that looks exactly like the active drug in terms of size and thickness. The drugs are commenced from 12 to 16 weeks' gestation and continued until 36 weeks or delivery, whichever comes earlier. Emzor Pharmaceutical Industries Limited, a reputable Nigerian pharmaceutical company, which 
currently manufacture $75 \mathrm{mg}$ aspirin, manufactured the active drugs and placebo.

\section{Adherence}

The women are sent daily reminders by text message, questioned on compliance at each visit and asked to bring in their used sachets for sighting. Extra drugs were produced to accommodate losses but in the unlikely event that they are all used up, the trial pharmaceutical company will be asked to produce a new pack of active or placebo drug.

\section{Concordant care}

Participants are being told to report adverse drug reactions early, and the cost of managing any adverse drug reaction attributable to the LDA will be borne by the investigators. Adverse events will be recorded on the adverse event case report form (CRF) as any symptom that starts or worsens after the study drugs have been commenced; the severity (mild, moderate, severe), relationship to the study drug (suspected/not suspected), duration (start and end dates or if continuing at final exam) and whether it constitutes a serious adverse event will also be recorded.

\section{Outcomes}

Primary outcome is the incidence of birth weight below 10th centile for gestational age on the WHO INTERGROWTH-21st birth weight charts, or stillbirth, or fetal death or miscarriage after randomization, as a composite outcome. Secondary outcome measures are $\mathrm{PE}$, maternal death, preterm delivery, perinatal death, number of crises, need for blood transfusion; complications such as urinary tract infection, lower respiratory tract infection, acute chest syndrome, retained placenta, placental abruption; and potential adverse effects such as vaginal bleeding, epigastric pain and heartburn.

PE will be diagnosed based on the International Society for the Study of Hypertension in Pregnancy classification $^{16}$ if there is development of hypertension (either systolic blood pressure $\geq 140 \mathrm{~mm} \mathrm{Hg}$ or diastolic blood pressure $\geq 90 \mathrm{~mm} \mathrm{Hg}$ on two occasions at least 4 hours apart) after 20 weeks' gestation plus one of the following: proteinuria, thrombocytopaenia or any multisystem complication. Proteinuria will be defined as a dipstick test with $1+$ proteinuria $(\geq 30 \mathrm{mg} / \mathrm{dL}$ ) on two occasions at least 4 hours apart, without any evidence of a urinary tract infection. If proteinuria of $\geq 1+(\geq 30 \mathrm{mg} / \mathrm{dL})$ is recorded in a participant with elevated blood pressure (hypertension), urine specimen will be collected for urinary protein to creatinine ratio estimation (UPCR) to confirm if proteinuria is significant and a value of $30 \mathrm{mg} / \mathrm{mmol}$ will be considered to be significant. Thrombocytopaenia will be defined as a platelet count of $<100000 / \mathrm{mm}^{3}$.

\section{Participants' timeline}

Eligible participants are being enrolled at 12-16 weeks gestational age and followed up until delivery (table 1).

\section{Sample size}

Assuming an incidence of IUGR of $20 \%$ in $\mathrm{SCD}^{17} 18$ and expecting a 50\% IUGR reduction with the use of LDA as detected by Bujold et al, ${ }^{19}$ we calculated that 198 women per group would be required to have an $80 \%$ power of detecting a decrease in IUGR at the $5 \%$ significance level, with a total of 396, using the formula for proportions in superiority parallel trials. ${ }^{20}$ Allowing for $20 \%$ attrition brings the total sample size to 476 .

\section{Recruitment}

The women are being recruited from the antenatal clinics of the hospitals with a purposive sampling method that is, consecutively as long as they meet the eligibility criteria. A screening log is being maintained at all study sites and includes details of all screened pregnant $\mathrm{HbSS}$ and $\mathrm{HbSC}$ women. Only those that meet the eligibility criteria are recruited and have their details entered in the enrollment $\log$.

\section{Randomisation and allocation}

This is accomplished by a web-based software, 'Sealed envelope' in a 1:1 ratio in blocks stratified according to site. Sealed envelope has generated a randomisation code list, shared only with the unblinded pharmacist by email. The code list is in blocks of four, each code is pasted on the appropriate drug kit and the drug kits are sent out in blocks of four to each site by the unblinded pharmacist.

Table 1 Study time frame

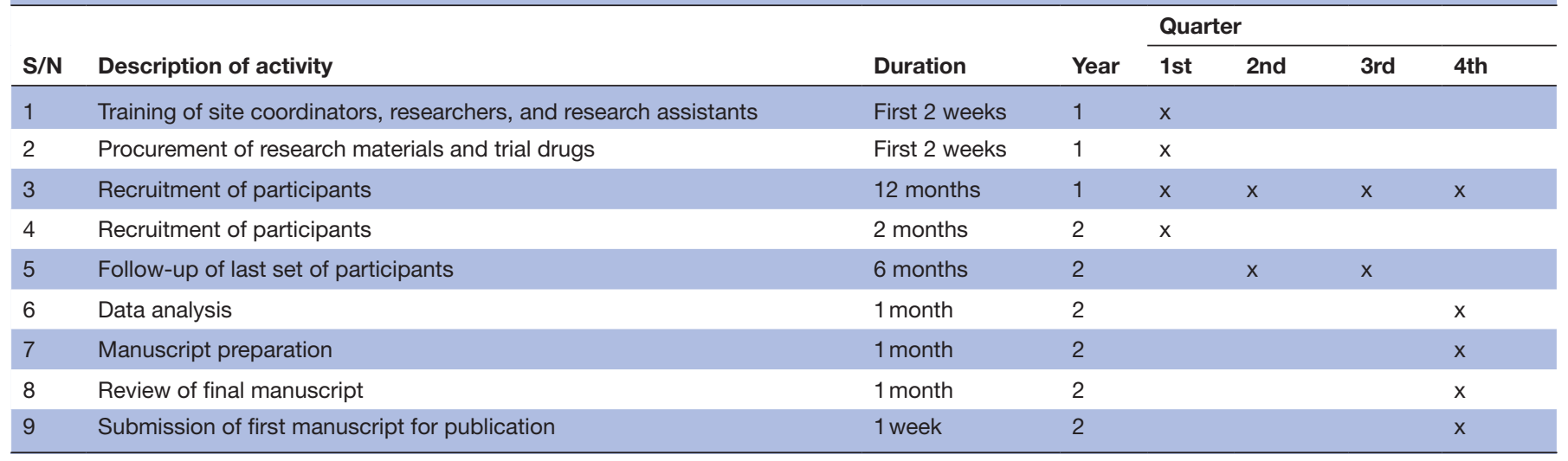


Once the drugs with their attached codes are received by each site, the site coordinator informs the Principal Investigator (PI), who loads the appropriate codes onto the system. As each new patient is recruited from a particular site, her details are entered into the randomisation software using an electronic hand-held computing device and a code is generated, which will be one of the codes that were sent to the site. She is then given tablets from the box of drugs that carries the generated code.

\section{Blinding}

Only an unblinded pharmacist, who is not in contact with the participants, is aware of the actual codes. Emergency unblinding will only happen if a participant suffers a severe adverse drug reaction that is adjudged to be life threatening and suspected to be related to aspirin.

\section{Data collection methods}

All site coordinators and their research nurses were trained on the trial protocol, research ethics and issues relating to protocol violation and deviation, prior to commencement of this randomised controlled trial (RCT). Once a participant is recruited, the recruitment number is retrieved from the joint database and written on her notes. The parcel corresponding to her recruitment number is opened and she is given a 2-week pack (or 4-week pack depending on the appointment interval) of the drugs within the parcel. The tablets are prepared in sachets and dispensed in 2-4 weekly doses.

Each woman who self-reports or is found to have SCD on routine haemoglobin genotype testing will have a confirmatory haemoglobin electrophoresis done. This is to ensure a uniform diagnosis and to be certain of the exact sickle cell phenotype. Every woman with HbSS or HbSC that fits the eligibility criteria and gives informed consent is consecutively recruited. Their haemoglobin fraction is assayed by High Performance Liquid Chromatography at enrolment. Their full blood count is also done at enrolment.

The women are seen at intervals of 2-4 weeks at the antenatal clinic up till 28 weeks and then weekly thereafter till delivery. We measure their body weight, blood pressures, haemoglobin concentration and urinalysis for protein and glucose at every visit. If proteinuria of $\geq 1+$ $(\geq 30 \mathrm{mg} / \mathrm{dL})$ is recorded in a participant with elevated blood pressure (hypertension), urine specimen will be collected for UPCR to confirm if proteinuria is significant and a value of $30 \mathrm{mg} / \mathrm{mmol}$ and above is considered to be significant. They have urinalysis done at booking and at every visit; and venous blood full blood count is done at booking and at delivery. Their oxygen saturation will be monitored in addition to other vital signs such as pulse rate, blood pressure, temperature and respiratory rates at every visit and whenever they are admitted to the hospital. At delivery, the babies will be weighed naked to determine if they have low birth weight based on the WHO INTERGROWTH-21st Chart. Following delivery, placental biopsies will be taken for histology to identify abnormalities that might be associated with some pregnancy complications such as PE and IUGR as well as the sickling process.

\section{Retention strategies}

The participants' phone numbers and addresses as well as phone numbers of their spouse and phone numbers and addresses of two close relatives will be recorded at enrolment. In the event that they do not attend their appointed clinic, the research nurse will send reminder text messages, make phone calls or visit them at home if necessary.

\section{Data management}

Data will be entered directly with assigned codes. The data file will be accessible by the PI and the statistician. Data will be captured in electronic CRFs (e-CRFs) at various patient visit types and uploaded real time to the central server after being checked by the site coordinator. They will follow the guidelines specified in the Standard Operating Procedure developed by the Data handling and communications committee for data collection, data entry and transmission, data compilation and management and data quality and security.

\section{Statistical methods}

Data analysis will be by intention-to-treat. Categorical variables will be expressed as frequencies and percentages. For continuous variables, a Shapiro-Wilk test of normality will be performed and normally distributed data will be presented as means $\pm 95 \%$ CI, while non-normally distributed data will be presented as median and IQR. It is not expected to have missing data in this study but in the event of missing data, it will be shown descriptively and bivariate analysis of the comparison of missing data among the intervention and control group will be conducted. If the percentage of missing values is more than $10 \%$, sensitivity analyses will be done based on multiple imputation of the missing data.

The risk of occurrence of IUGR, perinatal death and other key outcome variables will be computed and compared in both groups. A multivariate logistic regression analysis will be performed to determine the odds of each of the key outcomes among women who received the intervention with respect to those who did not, after controlling for common confounders. This will be presented as regression coefficients and their 95\% CI. Level of significance will be set at $5 \%$. Post-regression analysis will be performed to determine the goodness-of-fit of the final model. Two-tailed test of hypothesis will be assumed. Descriptive and bivariate analysis of the adherence pattern will be conducted. Sensitivity analysis will be performed to account for the potential for bias based on non-adherence to treatment by conducting propensity score matching and inverse probability weighting. STATA V.15.0 (StataCorp LP) or other suitable software will be used for statistical analysis. An interim analysis will be done after the 50th participant has completed the study. 


\section{Data monitoring}

Apart from the administrative core which consists of the PI and a full time project coordinator, other formal committees that will play key roles in monitoring the project are the Steering Committee (SC), Clinical Trial Monitors and the Data and Safety Monitoring Committee (DSMC). The Administrative Core provides administrative oversight and management of the research study and coordinates all internal and external meetings of investigators and staff.

The SC will ensure a successful delivery of the project, maximising the benefits from the projects and ensuring the approved methodology is followed. This committee will provide cross-functional leadership and direction, provide project management governance, and accept responsibility for the project strategy and the overall benefit realisation of the project. The SC will comprise the PI, all co-investigators, project site coordinators as may be appointed and a patient representative. The SC will monitor project progress by holding a bi-weekly on-line meeting (Zoom/Skype) to evaluate enrolment rate, challenges and issues as they arise. The committee will also hold a bi-annual face to face meeting to monitor and evaluate progress and ensure compliance with regulatory, fiscal and reporting requirements.

The clinical monitors are responsible for trial monitoring. Their role is to verify that the rights and well-being of human subjects are protected; that the reported trial data are accurate, complete and verifiable from source documents; and that the conduct of the trial is in compliance with the currently approved protocol/amendment(s), with Good Clinical Practice (GCP), and with the applicable regulatory requirement(s). They conducted pre-trial monitoring visits to ensure each centre and their site coordinators were ready; they delivered study material, documents and products and made sure the investigational team understood the protocol and GCP requirement. They will also conduct routine monitoring visits to make sure the study is conducted according to the protocol and GCP and help the investigational team in solving problems; and a close-out visit to make sure the investigator file is archived properly and collect back all unused material, documents or products.

Appointed members of the SC will be trained in GCP and research monitoring to perform the duties of clinical monitors. A site monitoring visit log will be maintained at the study site in which all visits made by authorised individuals are recorded. All clinical records and the e-CRFs for all the participants enrolled in this study will be made available for review to authorised individuals.

The DSMC is an independent external monitoring committee made up of three members including an obstetrician, a haematologist and a statistician. The committee members will meet at a minimum once before the study begins and every 6 months during the study.

An interim analysis will be done by the DSMC after the 50th participant has completed the study in order to assess progress of the RCT, recruitment rate, effect of drugs and decide whether or not to modify, terminate or continue the clinical trial. The monitoring teams shall ensure that the participants' rights are protected and that no patient suffers undue harm in the course of the RCT. Auditing of research records will be done by the monitoring committees as outlined above, while financial auditing will be done by the Research Management Office and Audit Department of University of Lagos.

\section{Patient and public involvement}

We interviewed five current and former pregnant women with SCD, asking about pregnancy problems, potential participation in such a study and advice on unaddressed issues. Feedback included concerns about whether the intervention had long term effects or could cause miscarriage. All the patients consented to participate if eligible and one of them attends monthly meetings of the study site coordinators. The results of the study will be communicated directly to the participants by the research nurses, physically as well as by email and Whatsapp messages.

\section{Ethics and dissemination \\ Research ethics approval}

Ethical approvals were granted by the Health Research and Ethics committees of the Lagos University Teaching Hospital, Idi-Araba (HREC No. ADM/DST/HREC/ APP/3301), Lagos State University Teaching Hospital, LASUTH (Ref.No. LREC/06/10/1318), Federal Medical Centre, Ebute Metta (Ref. No. FMCEB/ RET/0052) and the Health Service Commission of Lagos State for the use of the 12 General Hospitals within the state as study sites (LSHSC/2222/VOLIII). Approval has also been granted by the National Health Research and Ethics Committee (NHREC Approval No. NHREC/01/01/2007-04/12/2020).

\section{Consent and autonomy}

All participating women will sign the study's informed consent form prior to entry into the study. They will be told that they have the right to decide whether or not to partake in the trial and the freedom to withdraw during the course of the study if they so wish.

\section{Confidentiality}

All data will be kept strictly confidential. The site coordinator of each centre will collect all the data at his/her facility and send it to the principal investigator at periodic intervals. This will be stored securely in a central electronic database by the principal investigator who will be the only one with access to the data of all participants collated centrally. The statistician will be granted access to the electronic database during statistical analysis or at any other time the principal investigator might require her to review the data.

\section{Beneficence}

None of the women will be made to pay for any aspect of the study as the medication for the research will be given at no cost throughout the pregnancy. All investigations 
pertaining to this research will also be conducted at no cost to the participants.

\section{Non-maleficence}

This proposed research poses minimal or no risk to both mother and baby. Blood specimen collection might cause minimal discomfort in form of pain. For this reason, all the blood specimen collection will be made as comfortable as possible for the women.

\section{Justice}

The participants will enjoy equal rights and quality care all through the duration of the research.

\section{Access to data}

We will store the data and deposit it in 'Open Science Framework', after approval is obtained from the ethics committee. We will also provide metadata along with the data to describe it. Most of these data will have value to other research users but we will not share the identifying personal biodata such as home address, phone numbers or occupation. We will share the data at the time of publication of our first paper. We will share the assigned DOI number, the Open Science Framework website details and our approach to data sharing as an appendix to our publications to aid accessibility. We will also share these at any conference presentations and our study website, as well as local meetings and conferences of our Society of Obstetrics and Gynaecology.

\section{Dissemination}

The findings of this study will be presented at conferences (both international and local) so as to disseminate them to a large body of professionals in the field of Obstetrics and Gynaecology. We will also publish them in high impact peer reviewed journals for wider dissemination of information. The findings will be used in counselling pregnant women with SCD at the various antenatal clinics on complications associated with the disease and preventive measures that may be employed. Charts will be created to facilitate counselling at the various antenatal clinics. We will issue press releases about the findings of the study. Patients with SCD and members of the public were involved in the conduct and design of this study and will be included in its dissemination.

\section{Author affiliations}

${ }^{1}$ Obstetrics and Gynaecology, University of Lagos College of Medicine, Idi-Araba, Lagos, Nigeria

${ }^{2}$ Haematology and Blood Transfusion, University of Lagos College of Medicine, IdiAraba, Lagos, Nigeria

${ }^{3}$ Community Health and Primary Care, University of Lagos, Idi-Araba, Lagos, Nigeria ${ }^{4}$ Paediatrics, University of Lagos College of Medicine, Idi-Araba, Lagos, Nigeria

${ }^{5}$ Pharmacology, University of Lagos College of Medicine, Idi-Araba, Lagos, Nigeria

${ }^{6}$ Obstetrics \& Gynaecology, Lagos State University College of Medicine, Ikeja, Lagos, Nigeria

${ }^{7}$ Chemical Engineering, University of Delaware, Newark, Delaware, USA

\section{Twitter Bosede Bukola Afolabi @Coolgynae}

Acknowledgements We acknowledge the support of Tertiary Education Trust Fund (TETFund) of the Federal Government of Nigeria, Grant Number: TETFund/DR\&D/CE/ NRF/STI/28/NOL1, for funding this study. We acknowledge Emzor Pharmaceutical Ltd for supplying the main study drug - 100mg aspirin and placebo, free of charge.
We also acknowledge the study participants and all the patient contributors especially Mrs Bukola Durowoju and Mrs Ayoola Akinyeye, for their valuable advice and insight.

Collaborators Gbenga Olorunfemi.

Contributors BBA was involved in the conceptualisation of the research idea, drafted the first version of this protocol, led the team in applying for the grant to fund the research and supervised the drafting of the second version of the protocol. TAA, OAB, 000, YAO and BA0 contributed to the research idea. TAA, OAB, 000, YAO, VCE and ON contributed by drafting some sections of the first and second versions of this protocol and also contributed to the version submitted for funding. All authors reviewed and approved the final version of this protocol for publication.

Funding This work is being supported by the Tertiary Education Trust Fund (TETFund) of the Federal Government of Nigeria, Grant Number: TETFund/DR\&D/ CE/NRF/STI/28/VOL1. Contact address of funding agency: TETFund Building, № 6 Zambezi Crescent, Off Aguiyi Ironsi Street, Maitama, Abuja, FCT, Nigeria; Tel: (+234) 8008383863; https://www.tetfund.gov.ng.

Competing interests None declared.

Patient and public involvement Patients and/or the public were involved in the design, or conduct, or reporting, or dissemination plans of this research. Refer to the Methods section for further details.

Patient consent for publication Not required.

Provenance and peer review Not commissioned; externally peer reviewed.

Open access This is an open access article distributed in accordance with the Creative Commons Attribution Non Commercial (CC BY-NC 4.0) license, which permits others to distribute, remix, adapt, build upon this work non-commercially, and license their derivative works on different terms, provided the original work is properly cited, appropriate credit is given, any changes made indicated, and the use is non-commercial. See: http://creativecommons.org/licenses/by-nc/4.0/.

ORCID iD

Bosede Bukola Afolabi http://orcid.org/0000-0002-7511-7567

\section{REFERENCES}

1 WHO, Fifty-ninth World Health AssemblyA59/9. Sickle-cell anaemia report by the secretariat World Health Organisation; 2006a.

2 Chakravorty S, Williams TN. Sickle cell disease: a neglected chronic disease of increasing global health importance. Arch Dis Child 2015;100:48-53.

3 Yawn BP, Buchanan GR, Afenyi-Annan AN, et al. Management of sickle cell disease: summary of the 2014 evidence-based report by expert panel members. JAMA 2014;312:1033-48.

4 Rees DC, Williams TN, Gladwin MT. Sickle-cell disease. Lancet 2010;376:2018-31.

5 Oteng-Ntim E, Ayensah B, Knight M, et al. Pregnancy outcome in patients with sickle cell disease in the UK--a national cohort study comparing sickle cell anaemia (HbSS) with $\mathrm{HbSC}$ disease. $\mathrm{Br} \mathrm{J}$ Haematol 2015;169:129-37.

6 Villers MS, Jamison MG, De Castro LM, et al. Morbidity associated with sickle cell disease in pregnancy. Am J Obstet Gynecol 2008;199:125.e1-125.e5.

7 Afolabi BB, Iwuala NC, Iwuala IC, et al. Morbidity and mortality in sickle cell pregnancies in Lagos, Nigeria: a case control study. $J$ Obstet Gynaecol 2009;29:104-6.

8 Say L, Souza JP, Pattinson RC, et al. Maternal near miss--towards a standard tool for monitoring quality of maternal health care. Best Pract Res Clin Obstet Gynaecol 2009;23:287-96.

9 Obilade OA, Akanmu AS, Broughton Pipkin F, et al. Prostacyclin, thromboxane and glomerular filtration rate are abnormal in sickle cell pregnancy. PLoS One 2017;12:e0184345.

10 Lewis DF, Canzoneri BJ, Gu Y, et al. Maternal levels of prostacyclin, thromboxane, ICAM, and VCAM in normal and preeclamptic pregnancies. Am J Reprod Immunol 2010;64:376-83.

11 Duley L, Henderson-Smart DJ, Meher S, et al. Antiplatelet agents for preventing pre-eclampsia and its complications. Cochrane Database Syst Rev 2007;2.

12 Moore GS, Allshouse AA, Post AL, et al. Early initiation of low-dose aspirin for reduction in preeclampsia risk in high-risk women: a secondary analysis of the MFMU high-risk aspirin study. J Perinatol 2015;35:328-31.

13 RCOG. Management of sickle cell disease in pregnancy. In: Royal College of obstetricians and gynaecologists green-top guideline, 2011: 1-20. 
14 Nwabuko O, Okoh D, lyalla C, et al. Prevalence of sickle cell disease among pregnant women in a tertiary health center in South-South Nigeria. Sub-Saharan Afr J Med 2016;3:132-6.

15 Olugbenga A. Managing sickle cell disease in pregnancy, the success and the challenges: our experience in a semi-urban tertiary health-care facility, Southwest, Nigeria. Trop J Obstet Gynaecol 2018;35:342-7.

16 Brown MA, Lindheimer MD, de Swiet M, et al. The classification and diagnosis of the hypertensive disorders of pregnancy: statement from the International society for the study of hypertension in pregnancy (ISSHP). Hypertens Pregnancy 2001;20:ix-xiv.
17 Al Jama FE, Gasem T, Burshaid S, et al. Pregnancy outcome in patients with homozygous sickle cell disease in a university hospital, Eastern Saudi Arabia. Arch Gynecol Obstet 2009;280:793-7.

18 Sun PM, Wilburn W, Raynor BD, et al. Sickle cell disease in pregnancy: twenty years of experience at grady memorial hospital, Atlanta, Georgia. Am J Obstet Gynecol 2001;184:1127-30.

19 Bujold E, Roberge S, Lacasse Y, et al. Prevention of preeclampsia and intrauterine growth restriction with aspirin started in early pregnancy: a meta-analysis. Obstet Gynecol 2010;116:402-14.

20 Sealed Envelope Ltd. Power calculator for binary outcome equivalence trial. [Online], 2012. Available: https://www. sealedenvelope.com/power/binary-equivalence/ 